EPJ Web of Conferences 59, 14002 (2013)

DOI: $10.1051 /$ epjconf/20135914002

(C) Owned by the authors, published by EDP Sciences, 2013

\title{
Free-bound electron exchange contribution to l-split atomic structure in dense plasmas
}

\author{
K. Bennadji ${ }^{1,2, a}$, F. Rosmej ${ }^{1,3}$ and V.S. Lisitsa ${ }^{4}$ \\ ${ }^{1}$ Sorbonne Universités, Pierre et Marie Curie UPMC, LULI, UMR 7605, case 128, 4 place \\ Jussieu, 75252 Paris Cedex 05, France \\ ${ }^{2}$ ExtreMe Matter Institute EMMI, GSI, Planckstr. 1, 64291 Darmstadt, Germany \\ ${ }^{3}$ Ecole Polytechnique, Laboratoire pour l'Utilisation des Lasers Intenses LULI, Physique \\ Atomique dans les Plasmas Denses PAPD, 91118 Palaiseau, France \\ ${ }^{4}$ Russian Research Center, "Kurchatov Institute", Moscow 123182, Russia
}

\begin{abstract}
An analytical expression for the exchange energy between the bound electron in hydrogen-like ions and the free electrons of plasma is proposed. Two limiting cases are identified: 1) the low temperature limit where the energy depends linearly on density and on the ion charge as $1 / Z^{2}$ but does not depend on the temperature itself, 2) the high temperature limit where the energy depends on temperature as $1 / T$ but does not depend on the ion charge. These two regimes are separated by a characteristic temperature $\left(T^{*}=4 Z^{2} R y\right)$ which is a universal parameter depending only on the charge $Z$ of the ions. We presented numerical results for aluminum: the exchange energy contributes about $15 \%$ to the total plasma energy and can reach an order of $10^{-4}$ of the total transition energy. Comparison to the Local-density Approximation (Kohn-Sham) exchange energy shows a good agreement.
\end{abstract}

\section{INTRODUCTION}

Plasma-atom interactions are greatly needed in modeling problems involved with Inertial Fusion Confinement. Accurate evaluation of plasma properties such as opacity, equation of state etc. needs accurate knowledge of ingredients involved in dynamical equations such as Hartree-Fock or Dirac-Fock equations $[1,2]$. One of these ingredients is the exchange potential between a bound electron of an ion and the free electrons of the plasma. This potential is usually approximated in the Local-Densityapproximation (LDA) by a Kohn-Sham expression [3] and extending its validity to non-local densities. This expression was derived in the case of free electron gas and it seems to work also in the case of bound electrons by substituting the constant density of the free electrons by the variable density of bound electrons. The aim of this work is to derive accurate formulas from fundamental formalism involving symmetrization of the global state of all electrons including one bound electron in order to account for free-bound exchange. The bound electron wave function is the coulombic one [4], which allows us to incorporate atomic parameters together with plasma parameters. Exchange energy is obtained by using a differential method and the results can be generalized to many electron atoms by using an effective charge of a hydrogen-like ion.

\footnotetext{
ae-mail: kbennadji@hotmail.com
}

This is an Open Access article distributed under the terms of the Creative Commons Attribution License 2.0, which permits unrestricted use, distribution, and reproduction in any medium, provided the original work is properly cited. 


\section{DIFFERENTIAL METHOD FOR EXCHANGE-ENERGY EVALUATION}

Our system is constituted by one bound electron and $N-1$ free electrons. The excess Coulomb energy due to the bound electron can be evaluated by the difference between a total energy including the bound electron and a total energy where the bound electron is not included (differential method):

$$
E_{c}=\left\langle\Phi_{N}\left|\Phi_{N}\right| \Phi_{N} \mid\right\rangle-\left\langle\Phi_{N-1}\left|\Phi_{N-1}\right| \Phi_{N-1}\right\rangle
$$

The label $N-1$ refers to a system of $N-1$ free electrons and the label $N$ to a system of $N-1$ free electrons plus one bound electron. $\left|\Phi_{N^{\prime}}\right\rangle$ is antisymmetric in the exchange of positions and spin:

$$
\left|\Phi_{N^{\prime}}\right\rangle=\frac{1}{\sqrt{N^{\prime} !}} \sum_{P}^{N^{\prime} !} \varepsilon_{p}\left|\varphi_{p_{1}} \ldots \ldots \ldots \varphi_{P_{N^{\prime}}}\right\rangle, \quad N^{\prime}=N \text { or } N-1
$$

Among the $N$ individual states in (1), one of them is a bound state described by a coulombic wave function, other states are plane waves. The potential operator in (1) is:

$$
\Phi_{N^{\prime}}=\sum_{i=1}^{N^{\prime}} \sum_{\substack{j=2 \\
j>i}}^{N^{\prime}} \frac{e^{2}}{\left|\begin{array}{lr}
\mathrm{r} & \mathrm{r} \\
\mathrm{r}_{i}-\mathrm{r}_{j}
\end{array}\right|}, \quad N^{\prime}=N \text { or } N-1 .
$$

Because of the symmetrization (2), expression (1) will split into two terms, a direct term and an exchange term. Calculation of the latter gives:

$$
E_{e x}=-\sum_{k=2}^{N} \operatorname{Re}\left[\left\langle\varphi_{1} \varphi_{k}\left|\frac{e^{2}}{\left|\begin{array}{rr}
\mathrm{r} & \mathrm{r} \\
\mathrm{r}_{1}-\mathrm{r}_{2}
\end{array}\right|}\right| \varphi_{k} \varphi_{1}\right\rangle\right]
$$

The index 1 stands for the bound state and other indexes for the plane waves. Additional calculations give a general expression for the exchange energy at arbitrary degeneracy and density:

$$
\begin{aligned}
E_{e x}= & -\frac{e^{2} k_{F}^{2} N_{l m}^{2} a_{0}}{\pi Z} \int_{0}^{\pi} d \varphi \cos (m \varphi) \int_{-1}^{+1} d u_{1} P_{l}^{m}\left(u_{1}\right) \int_{-1}^{+1} d u_{2} P_{l}^{m}\left(u_{2}\right) \int_{0}^{\infty} d x_{1} x_{1}^{2} F_{n l}\left(x_{1}\right) \\
& \times \int_{0}^{\infty} d x_{2} x_{2}^{2} F_{n l}\left(x_{2}\right) \frac{1}{x^{2}} \int_{0}^{\infty} f_{F D}(y) \sin (A x y) .
\end{aligned}
$$

Non-dimensional quantities are used; see references $[4,5]$ for definitions. $x$ is the distance between two electrons, $x_{1}$ and $x_{2}$ are the positions. Temperature is included through Fermi-Dirac distribution.

The parameter $A\left(A=n a_{0} k_{F} / 2 Z\right)$ is of great importance. It links between atomic scaling and plasma scaling. $Z$ is an effective charge felt by the bound electron (eventually, other bound electrons can be taken into account by this effective charge). The parameter $A$ follows from the use of the coulombic wave function for the bound electron. It is worth noticing that if we use a plane wave instead of the coulombic wave function, we will obtain (at zero temperature) exactly the result of Kohn-Sham [3] for free electron gas; see formula (10) below. The use of bound wave function allows us to incorporate atomic parameters $(Z, n, l)$ in addition to the plasma parameters $\left(n_{e}, T\right)$ in the exchange energy. 
Table 1. Comparison between exchange effect and plasma effect in helium-like aluminum at electron density of $2.8 \times 10^{22} \mathrm{~cm}^{-3}$ and temperature of $70 \mathrm{eV}$.

\begin{tabular}{|l|l|}
\hline Total $1 s 2 p \rightarrow 2 s^{2}$ energy transition $(\mathrm{eV})$ & 1593 \\
\hline Free-bound exchange energy contribution to line shift $(\mathrm{eV})$ & 0.06 \\
\hline Total plasma contribution to the line shift [6] (eV) & 0.4 \\
\hline
\end{tabular}

\section{LOW TEMPERATURE LIMIT (LTL)}

At low temperatures, $T \ll T^{*}$ where $T^{*}$ is a characteristic temperature to be defined below in (9), the formula (2) reduces to:

$$
E_{e x}^{0}=-\frac{e^{2} a_{0}^{2} k_{F}^{3}}{3 \pi} \frac{n}{(2 l+1) Z^{2}} \int_{0}^{\infty} d x_{1} x_{1}^{1-l} F_{n l}\left(x_{1}\right) \int_{0}^{x_{1}} d x_{2} x_{2}^{2+l} F_{n l}\left(x_{2}\right) .
$$

Expression (6) is easy to calculate. After calculations for some varying values of $n$ and $l$, we deduce a fit formula:

$$
E_{e x}^{0}\left(Z, n, l, n_{e}\right)=-B \frac{n^{3.28}}{(2 l+1) Z^{2}}\left[1-\frac{6 l}{8 n-3}\right] n_{e} ; \quad B=2,5417 \times 10^{-22} \mathrm{eV} \mathrm{cm}^{3} .
$$

Expression (7) is very convenient to use as a modeling of the bound-free exchange potential. Exchange energy depends on four parameters and is not sensitive to the temperature. The validity domain is very large and it covers many laser experiment domains.

\section{HIGH TEMPERATURE LIMIT (HTL)}

For non-degenerate case, $f_{F D}$ becomes a maxwellian distribution and eq. (5) simplifies to:

$$
\begin{aligned}
E_{e x}= & -\frac{2^{10} e^{2} N_{l m}^{2} Z^{3}}{3 \pi k_{F}^{2} a_{0}^{3} n^{4} \Theta^{-5 / 3}} \int_{0}^{\pi} d \varphi \cos (m \varphi) \int_{0}^{+1} d u_{1} P_{l}^{m}\left(u_{1}\right) \int_{-1}^{+1} d u_{2} P_{l}^{m}\left(u_{2}\right) \int_{0}^{\infty} d x_{1} x_{1}^{2} F_{n l}\left(\lambda x_{1}\right) \\
& \times \int_{0}^{\infty} d x_{2} x_{2}^{2} F_{n l}\left(\lambda x_{2}\right) \frac{e^{-x^{2}}}{x}
\end{aligned}
$$

where $\lambda$ is the ratio of thermal De-Broglie wavelength per atom mean radius. A thermal De-Broglie sphere is surrounding the bound electron. The exchange energy takes non-vanishing values for each free electron inside the sphere and drops exponentially for electrons outside the sphere.

In order to reproduce the LTL and the HTL, a crude modeling is introduced. This one assumes that exchange operates inside a sphere of radius $R$ surrounding the bound electron so that the exchange takes significant values for each free electron inside the sphere and vanishes for electrons outside. In the LTL, the radius is simply the atom radius $\left(R_{a}=n a_{0} / Z\right)$ and in the HTL, the radius is the thermal De-Broglie radius. The limiting case between the two radii gives a characteristic temperature:

$$
T^{*}=54.4 Z^{2}(\mathrm{eV})
$$

\section{NUMERICAL RESULTS}

Table 1 compares between exchange effect, plasma effect and total transition energy in helium-like aluminum ion in plasma. The exchange energy contributes about $10^{-4}$ of the transition and about $15 \%$ of the total plasma shift. 

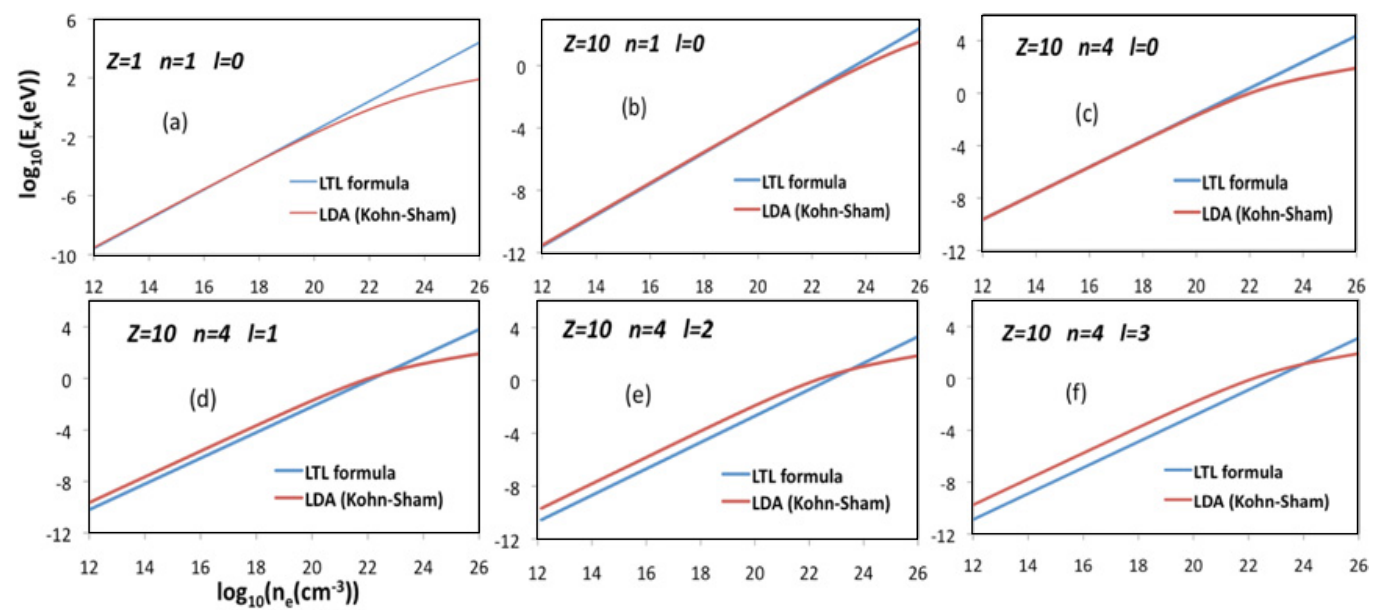

Figure 1. Exchange energy versus electron density in hydrogen-like ions $(Z=1,10)$. Comparison between LTL fit (formula (7)) and LDA (formula (11)).

Exchange energy has been obtained at zero temperature [3] and finite temperature [7, 8]. The KohnSham formula [3] is:

$$
\varepsilon_{e x}^{K S}=-e^{2}(3 / \pi)^{1 / 3} n^{1 / 3} .
$$

Formula (10) is established for uniform electron gas and Local density approximation (LDA) consists in extending this formula to a non-uniform electron gas by replacing the constant density by the varying one [9]. Doing so and multiplying by the density one obtains exchange energy per unit volume, integrating in finite volume gives exchange energy in that volume. We apply the same method as in (1) (differential method) to obtain the exchange energy of the bound electron in LDA:

$$
E_{e x}^{K S}\left(Z, n, l, n_{e}\right)=-4 \pi e^{2}(3 / \pi)^{1 / 3} \int_{0}^{\infty} d r r^{2}\left[\left(n_{b}(r)+n_{e}\right)^{4 / 3}-n_{b}^{4 / 3}(r)-n_{e}^{4 / 3}\right] .
$$

Results of LDA (11) and LTL fit (7) are plotted together in figures 1-a-f, for different values of charge number, principal quantum number and orbital quantum number. From these figures we can deduce:

- exchange energy from the present calculations and from Kohn-Sham expression (11) vary linearly with the density.

- both energies are close at low and intermediate densities for orbital momentum zero.

- exchange energy of the present calculations decreases with increasing orbital momentum while the one of LDA remains constant.

\section{CONCLUSION}

Free-bound electron exchange energy is calculated using a symmetrization procedure, taking atomic and plasma parameters into account. The result is a generalization of the Kohn-Sham one to bound states. Low and High temperature limits are obtained and a fit formula for low temperatures is deduced. Comparison of exchange energy to line shift due to plasma shows the importance of free-bound exchange. The fit formula is compared to the LDA values and a good agreement is revealed at low and intermediate densities. This agreement disappears gradually as the orbital momentum increases. 


\section{IFSA 2011}

\section{References}

[1] T. Blenski and K. Ishikawa, Phys. Rev. E 51, 4869 (1995)

[2] R. Piron and T. Blenski, Phys. Rev. E 83, 026403 (2011)

[3] W. Kohn and L. J. Sham, Phys. Rev. 140, A1133 (1965)

[4] F. Rosmej, K. Bennadji and V. S. Lisitsa, Phys. Rev. A 84, 032512 (2011)

[5] K. Bennadji, M.-M. Gombert and A. Bendib, Phys. Rev. E 79, 016408 (2009)

[6] O. Renner, P. Adamek et al. JQSRT 99, 532 (2006)

[7] S. Ichimaru, H. Iyetomiand, S. Tanaka, Phys. Rep. 149, 91 (1987)

[8] F. Perrot, Phys. Rev. A 20, 586 (1979)

[9] F. Perrot and M. W. C. Dharma-wardana, Phys. Rev. A 30, 2619 (1984) 